\title{
A etnobotânica e as plantas medicinais na Comunidade Sucuri, Cuiabá, MT, Brasil
}

The ethnobotany and medicinal plants in Community Sucuri, Cuiabá, MT, Brazil

L'ethnobotanique et plantes médicinales dans Communauté Sucuri, Cuiabá, MT, Brésil

La etnobotánica y plantas medicinales en Comunidad Sucuri, Cuiabá, MT, Brasil

\author{
Karina Gondolo Gonçalves* \\ (gondolo.karina@gmail.com) \\ Maria Corette Pasa** \\ (pasamc@brturbo.com.br)
}

Recebido em 02/04/2015; revisado e aprovado em 24/05/2015; aceito em 22/07/2015 DOI: http://dx.doi.org/10.1590/1518-70122015201

\begin{abstract}
Resumo: Objetivou-se investigar e analisar sistematicamente o conhecimento empírico dos moradores da Comunidade Sucuri em Cuiabá, MT, sobre a utilização dos recursos vegetais, ressaltando a finalidade e suas formas de usos. A pesquisa qualitativa e quantitativa utilizou o Pré-teste, Observação direta, Turnê guiada, Entrevistas, Frequências absoluta e relativa. Identificou-se 201 espécies vegetais em 36 quintais visitados. Foram citadas também 54 espécies medicinais retiradas da flora local.

Palavras-chave: Saber local. Recursos vegetais. Categorias de uso.

Abstract: This study aimed to investigate and analyzing empirical knowledge of the residents of Sucuri Community in Cuiabá, MT, on the use of plant resources, highlighting the purpose and forms of uses. Qualitative and quantitative research used the pre-test, Direct observation, guided tour, Interviews, absolute and relative frequencies. It identified 201 plant species visited by 36 yards. They were also cited 54 medicinal species taken from the local flora. Key words: Local knowledge. Plant resources. Use categories .

Résumé: Cette étude visait à examiner et analyser la connaissance empirique des résidents de Sucuri Communauté à Cuiabá, MT, sur l'utilisation des ressources végétales, en soulignant le but et les formes d'utilisations. La recherche qualitative et quantitative a utilisé le pré-test, observation directe, visite guidée, Interviews, fréquences absolues et relatives. Elle a identifié 201 espèces de plantes visitées par 36 verges. Ont également été cité 54 espèces médicinales prises de la flore locales.

Mots-clés: Les connaissances locales. Ressources végétales. Catégories d'utilisation

Resúmen: Este estudio tuvo como objetivo investigar y analizar el conocimiento empírico de los residentes de Sucuri Comunidad en Cuiabá, MT, en el uso de los recursos vegetales, destacando el propósito y las formas de usos. La investigación cualitativa y cuantitativa utiliza el pre-test, la observación directa, visita guiada, Entrevistas, frecuencias absolutas y relativas. Se identificaron 201 especies de plantas visitadas por 36 yardas. También fueron citadas 54 especies medicinales tomados de la flora local.

Palabras clave: conocimiento local. Recursos vegetales. Categorías utiliza.
\end{abstract}

\section{Introdução}

A etnobotânica é classicamente definida como a ciência que estuda o conhecimento e as conceituações desenvolvidas por qualquer sociedade a respeito do mundo vegetal, englobando tanto a maneira como o grupo social classifica as plantas, como a finalidade que lhes dá (AMOROZO, 1996).

Nessa percepção, a Etnobotânica passa a existir como sendo o campo interdisciplinar que compreende o estudo e a interpretação do conhecimento, significação cultural, manejo e usos tradicionais dos elementos da flora (CABALLERO, 1979).
Como ferramenta da etnobotânica, o saber local compreende em um sistema de classificação, uma série de observações empíricas do meio ambiente local, um sistema autônomo organizacional com fortes raízes no passado, um conhecimento ecológico acumulativo e dinâmico, o qual é construído sob a experiência de antepassados (JOHNSON, 1992).

Assim as observações populares sobre o uso e a eficácia de plantas medicinais contribuem de forma relevante para a divulgação das virtudes terapêuticas dos vegetais, prescritos com frequência, pelos efeitos medicinais que produzem, apesar de não te-

\footnotetext{
* Universidade Federal de Mato Grosso, Cuiabá, Mato Grosso, Brasil. Bolsa CAPES.

** Universidade Federal de Mato Grosso, Cuiabá, Mato Grosso, Brasil.
} 
rem seus constituintes químicos conhecidos. Dessa forma, usuários de plantas medicinais de todo o mundo, mantém em voga a prática do consumo de fitoterápicos, tornando válidas informações terapêuticas que foram sendo acumuladas durante séculos (COSTA; SILVA, 2014).

Para a Organização Mundial de Saúde (OMS), as plantas consideradas como medicinais precisam conter, em um ou mais de seus órgãos, substâncias que possam ser usadas com propósitos terapêuticos ou que sejam precursoras de síntese de fármacos (WHO, 1979).

Uma das mais importantes unidades de paisagem utilizadas nos estudos etnobotânicos são os quintais, tanto rurais como urbanos, por ser um local rico em diversidade vegetal e de conservação da biodiversidade. O quintal, de uma forma geral, pode ser compreendido como um espaço de usos múltiplos que fica próximo à residência do grupo familiar (AMOROZO, 2008).

De acordo com Pasa (2004, 2011), a produção nos quintais conserva não só os recursos vegetais como também a riqueza cultural, fundamentada no saber e na cultura dos moradores locais.

Corroborando com as afirmações acima, observa-se, nos dias atuais, uma grande evasão da população jovem pertencente às comunidades tradicionais ou de saberes locais para os grandes centros, em busca de melhores oportunidades de estudo ou de trabalhos, fazendo com que a perpetuação da cultura, dos costumes e saberes locais caminhem para a extinção. É possível que, em alguns anos, só se ouvirá falar de certas culturas em livros e registros.

O presente estudo tem por finalidade investigar e analisar sistematicamente o conhecimento empírico dos moradores da comunidade Sucuri em Cuiabá, MT, sobre a utilização das plantas da vegetação local, ressaltando a finalidade e suas formas de uso com intuito de resgatar o saber local dessa população.

\section{Metodologia}

O estudo foi realizado na Comunidade Sucuri, que é considerada politicamente como Distrito do município de Cuiabá e encontra- se circunscrita nas coordenadas geográficas latitude $15^{\circ} 32^{\prime} 50^{\prime \prime}$ Sul e longitude $56^{\circ} 09^{\prime} 26^{\prime \prime}$ Oeste, situada na porção Centro-Sul do Estado de Mato Grosso, estabelecido à margem esquerda do Rio Cuiabá. Altitude média correspondente é de 125 metros. Possui clima tropical semiúmido, apresentando duas estações bem definidas: uma seca (outono-inverno) e outra chuvosa (primavera-verão), predominando altas temperaturas, com médias de 24 a $34^{\circ} \mathrm{C}$ e de acordo com a classificação $\mathrm{Aw}$ Köppen (IBGE, 2010).

A pesquisa abordou uma análise qualitativa que, segundo Minayo (2007), leva em consideração os níveis mais profundos das relações sociais, operacionalizando-os através dos aspectos subjetivos, visando compreender a lógica interna de grupos, instituições e atores quanto aos valores culturais e representações sobre sua história.

Os sujeitos participantes da pesquisa foram os moradores da Comunidade Sucuri que atenderam aos seguintes critérios de inclusão: ambos os sexos, maiores de 18 anos de idade e que, após a explicação do objetivo do estudo, aceitaram de livre e espontânea vontade dela participar, assinando o Termo de Consentimento Livre e Esclarecido (TCLE).

Para a coleta dos dados socioeconômicos e etnobotânicos, foram utilizadas as técnicas metodológicas como o Pré-teste, Observação direta, Turnê guiada, Entrevistas dos tipos semiestruturada e aberta (MINAYO, 1994). As Entrevistas ocorreram entre os meses de julho a dezembro de 2014 totalizando 44 informantes residentes em 36 casas da comunidade local. As perguntas norteadoras da entrevista sobre os dados socioeconômicos foram: idade, sexo, religião, atividade profissional, escolaridade, renda familiar, estado civil, cor, número de pessoas na família, entre outras. Foram abordadas ainda questões referentes ao estado e hábitos de saúde, sobre o imóvel e quintal e, por fim, os dados etnobotânicos, que resgatam a relação do ser humano com as plantas na unidade de paisagem quintal e as diversas etnocategorias de usos pela população local.

O registro das entrevistas foi realizado por meio de um gravador digital portátil complementado por um diário de campo que tem por finalidade registrar as informações e acontecimentos pertinentes à pesquisa, sendo 
uma importante ferramenta usada durante a coleta de dados. Também foi utilizada uma máquina fotográfica digital para armazenar imagens que envolvem o manejo com as plantas, bem como dos diferentes equipamentos e materiais in loco.

Os nomes científicos e suas respectivas famílias botânicas foram classificados de acordo com o sistema de taxonomia vegetal APG III (APG, 2009).

\section{Resultados e discussão}

\subsection{Dados socioeconômicos}

Foram entrevistados 44 indivíduos, moradores das 36 residências visitadas, sendo a maioria do sexo feminino (77\%), que se justifica pelas atividades do lar e assim proporcionando a permanência da mulher na residência. Siveiro et al. (2014), em sua pesquisa realizada em três bairros da cidade de Rio Branco, no Acre, nos anos de 2009 a 2011, também mencionaram que maioria dos entrevistados foi do gênero feminino e casada. Esses dados são semelhantes também para muitas pesquisas realizadas em Mato Grosso porque, geralmente, as mulheres se ocupam com as tarefas do lar e os cuidados com os filhos, por isso passam mais tempo em casa e apresentam maior disponibilidade para participar da pesquisa.

A cor da pele não teve diferença significativa obtendo um resultado de $38,5 \%$, $34,1 \%$ e $27,5 \%$ respectivamente para negros, brancos e pardos.

A idade entre os informantes variou de 25 a 84 anos, tendo como representante da idade mais avançada o sexo masculino. No estudo de Almeida; Pasa; Guarim (2014) realizado em 2011, nas Comunidades Tradicionais da região de Barão de Melgaço, Estado de Mato Grosso, obtiveram-se resultados semelhantes tendo como faixa etária dos informantes a variação entre 33 e 85 anos de idade.

A religião católica obteve $57 \%$, seguida da evangélica, com aproximadamente $30 \%$, e a espírita, com quase $10 \%$, e dois homens relataram não possuírem religião.

Em relação ao estado civil dos depoentes, houve um destaque para os casados totalizando 23 indivíduos, os viúvos são representados por sete pessoas, os outros cinco referiram ser viúvos do primeiro casamento, porém hoje vivem amasiados com outro cônjuge, três mencionam ser solteiros e dois são divorciados.

O grau de instrução variou de não alfabetizado até nível superior, com ênfase para ensino fundamental completo, com aproximadamente $30 \%$, e ensino médio completo, com $25 \%$. O Estado de Mato Grosso foi o estado de origem que mais sobressaiu entre os moradores, com $86,5 \%$, sendo que muitos são de origem do próprio Distrito Sucuri. Entre outros Estados de origem, foram mencionados Mato Grosso do Sul, Paraná e Paraíba.

O tempo de residência no Distrito Sucuri teve uma variação de dois meses até 76 anos. A atividade exercida atualmente por essa população apresentou $34,10 \%$ para a classe dos aposentados e 13,60\% do lar e, classificados como outros, estão: comerciantes, cabeleireira, costureira, agente de saúde, pescador, merendeira etc. $\mathrm{O}$ estudo realizado no Acre por Siveiro et al. (2014) também apontou os 'aposentados' e as 'donas de casa' como as principais categorias de ocupação. A renda familiar que mais se destacou foi a dos que recebem entre um a três salários mínimos com um percentual de $68,20 \%$, seguido dos que recebem mais que três salários com $22,7 \%$, e os que sobrevivem com até um salário são representados por $9,1 \%$.

Referindo-se ao estado de saúde desses informantes e seus hábitos, pode-se identificar, através das informações emitidas pelos depoentes, que $61 \%$ deles possuem algum tipo de doença crônica, como diabetes, hipertensão arterial, hipotireoidismo, entre outras. Do total de entrevistados, 79,5\% não praticam nenhuma atividade física e também não fazem uso de cigarros, e $61 \%$ possuem planos de saúde, entre os quais o mais citado foi a Plano de Aposentadoria Complementar (PAC), que não tem cobertura total, mas sim um auxílio em consultas e exames.

Das 36 residências da comunidade local, a maioria é de alvenaria (95\%), sendo duas casas de madeira. Quanto à aquisição do imóvel, a maioria dos depoentes referiu morar em imóvel próprio, sendo que alguns disseram adquiri-los como herança de família. Um informante relatou morar de favor na casa de uma irmã, e outro mencionou residir na casa dos pais, e por fim um citou que mora 
de aluguel. A quantidade de peças variou entre três a dez cômodos. A energia elétrica e a água encanada estão presentes em 100\% das residências, e o Distrito Sucuri como um todo não possui rede de esgoto, e sim sistema de fossa séptica.

\subsection{Dados etnobotânicos dos quintais}

Os quintais são uma das formas mais antigas de manejo da terra que persiste até os dias atuais, pode-se dizer que essa tradição vem sendo passada de geração em geração. Para Siviero et al. (2014), os quintais residenciais são espaços de fácil acesso no qual os moradores cultivam uma diversidade de espécies vegetais com diversas funções de uso, como por exemplo: ornamentação, alimentação, medicação entre outros.

Os sistemas de quintais agroflorestais são conhecidos também como hortos caseiros onde ocorre o uso da terra na qual várias espécies de árvores são cultivadas junto com culturas perenes e anuais e, ocasionalmente, criação de pequenos animais ao redor da casa (DE DAVI et al., 2015).

Neste estudo, foi registrado um total de 201 espécies nos quintais da comunidade local, que estão distribuídas em 73 famílias botânicas e com um número de 992 citações para as espécies vegetais presentes nos quintais das residências locais. As espécies mais citadas foram: Mangifera indica L. (manga), Citrus limonum Risso. (limão), Cocos nucifera L. (coco), Malpighia glabra L. (acerola), Psidium guayava L. (goiaba), Allium fistulosum L. (cebolinha), Carica papaya L. (mamão), Anacardium occidentale L. (caju) e Musa sp. (banana). As famílias botânicas mais citadas são: Anacardiaceae com 91 espécies, Rutaceae com 80 e Lamiaceae com 62. Do total das 73 famílias, 15 foram citadas uma única vez, sendo Apocynaceae, Balsaminaceae, Burseraceae, Caprifoliaceae, Caricaceae, Compositae, Ericaceae, Nyctaginaceae, Orchidaceae, Polygonaceae, Portulacaceae, Punicaceae, Sapindaceae, Umbelliferae, Violaceae. Corroborando com os resultados, Almeida, Pasa e Guarim (2014) também apontaram a família Anacardiaceae, sendo a que apresentou maior riqueza de espécies nos quintais estudados na Comunidade de Cuiabá Mirim, Barão de Melgaço, Mato Grosso.
Os quintais do Distrito Sucuri são bastante variados tanto em tamanho como nas formas, quantidade e a variedade de espécies vegetais presentes nessa unidade de paisagem. Os quintais não foram mensurados, porém pode-se perceber a existência de quintais de todos os tipos desde os que circunvizinham toda a casa (frente, lateral e fundo) até os que ocupam somente uma das partes. Existem quintais limitados por muros e cercas, e também aqueles cujo limite é a própria vegetação nativa. Encontram-se ainda terrenos compartilhados, os quais antigamente eram de uma chácara, que, após herdarem dos pais, foi repartida entre os irmãos ou os filhos, que casaram e construíram suas casas próximas à dos pais, dividindo assim espaço no mesmo lote.

A quantidade de espécies vegetais presentes nos 36 quintais variou entre oito e 55 espécimes, com uma média de 23 espécies vegetais por quintal, nas residências visitadas. Como a maioria dos entrevistados é do sexo feminino, as mulheres são responsáveis pelos cuidados com o quintal. Quanto à frequência dispensada aos cuidados com o quintal, a maioria trabalha todos os dias.

A maioria dos depoentes informou que o principal destino das folhas e capinas é a compostagem, que serve de adubo para as plantas. Alguns depoentes referiram amontoar as folhas e depois, quando estiverem em fase de decomposição, as utilizam, e outros fazem buracos e as enterram usando esta terra para plantar.

A seca foi a época do ano relatada pela maioria dos entrevistados como sendo a mais trabalhosa para cuidar dos quintais, isto devido ao sol muito forte e à falta de chuva. Quanto ao uso de insumos, 73\% não fazem, utilizam nem compram, mas desenvolvem o adubo orgânico, através de métodos e produtos naturais, como terra preta e esterco de animais.

Conforme exposto na Tabela 1 , as etnocategorias de uso são: alimentar, medicinal, ornamental e outros. A mais expressiva foi a medicinal, com aproximadamente $42 \%$; em seguida, a alimentar com $25 \%$, a ornamental com $17 \%$ e $7 \%$ o total da categoria nomeada como outros, em que se inclui sombreamento, lenha, construção e, ainda, 9\% representando classe das espécies com mais de uma etnocategoria de uso. 
Tabela 1 - Plantas nos quintais da Comunidade Sucuri. Distrito Sucuri, Cuiabá, MT. 2014.

\begin{tabular}{|c|c|c|c|c|}
\hline Nome Popular & Nome Científico & Família & $\begin{array}{l}\text { Categoria } \\
\text { de Uso }\end{array}$ & NC \\
\hline manga & Mangifera indica L. & Anacardiaceae & $\mathrm{A}, \mathrm{M}$ & 40 \\
\hline limão & Citrus limonum Risso. & Rutaceae & A, M & 31 \\
\hline coco & Cocos nucifera L. & Arecaraceae & $\mathrm{A}, \mathrm{M}$ & 28 \\
\hline acerola & Malpighia glabra L. & Malpighiaceae & A, M & 27 \\
\hline goiaba & Psidium guayava L. & Myrtaceae & $\mathrm{A}, \mathrm{M}$ & 27 \\
\hline cebolinha & Allium fistulosum $\mathrm{L}$. & Alliaceae & A & 25 \\
\hline mamão & Carica papaya L. & Caricaceae & A, M & 24 \\
\hline caju & Anacardium occidentale L. & Anacardiaceae & A & 23 \\
\hline banana & Musa sp. & Fabaceae & A, M & 20 \\
\hline boldo do Chile & Plectranthus barbatus Andrews & Lamiaceae & M & 18 \\
\hline laranja & Citrus aurantium L. & Rutaceae & $\mathrm{A}, \mathrm{M}$ & 18 \\
\hline orquídea & Cattleya sp., Oncidium sp. & Orchidaceae & $\mathrm{O}$ & 18 \\
\hline samambaia & Nephrolepis exaltata (L.) Schott & Polypodiaceae & $\mathrm{O}$ & 18 \\
\hline mandioca & Manihot esculenta Crantz. & Euphorbiaceae & A & 17 \\
\hline cidreira ou capim-santo & Cymbopogon citratus (DC.) Stapf. & Poaceae & M & 16 \\
\hline camomila & Chamomilla recutita $\mathrm{L}$. Rauschert. & Asteraceae & M & 14 \\
\hline coentro & Coriandrum sativum $\mathrm{L}$. & Umbelliferae & A & 14 \\
\hline arruda & Ruta graveolens L. & Rutaceae & M & 13 \\
\hline jabuticaba & Myrciaria jaboticaba (Vell.) Berg & Myrtaceae & A & 13 \\
\hline abacate & Persea americana Mill. & Lauraceae & A, M & 11 \\
\hline $\begin{array}{l}\text { ata ou pinha ou } \\
\text { fruta-do-conde }\end{array}$ & Annona squamosa $\mathrm{L}$. & Annonaceae & A & 11 \\
\hline babosa & Aloe Vera (L.) Burm. F. & Liliaceae & M & 11 \\
\hline cajá-manga & Spondias dulcis Forst.F. & Anacardiaceae & A & 11 \\
\hline espada-de-são-jorge & $\begin{array}{l}\text { Sansevieria trifasciata var. } \\
\text { laurentii (Dewild.) N. E. Br. }\end{array}$ & Liliaceae & $\mathrm{O}$ & 11 \\
\hline hortelã & Mentha sp. & Lamiaceae & M & 11 \\
\hline jaca & Artocarpus heterophyllus Lam. & Moraceae & A & 11 \\
\hline romã & Punica granatum $\mathrm{L}$. & Punicaceae & $\mathrm{A}, \mathrm{M}$ & 11 \\
\hline seriguela ou jacote & Spondias purpurea L. & Anacardiaceae & A & 11 \\
\hline abacaxi & Ananas comosus (L.) Merril & Bromeliaceae & A & 10 \\
\hline bocaiúva & $\begin{array}{c}\text { Acrocomia aculeata (Jacq.) Lodd. } \\
\text { Ex mart. }\end{array}$ & Arecaraceae & A, M & 10 \\
\hline pimenta & Capsicum sp. & Solanaceae & A & 10 \\
\hline caninha-do-brejo & Costus spicatus (Jacq.) Sw. & Costaceae & M & 9 \\
\hline noni & Morinda citrifolia L. & Rubiaceae & M & 9 \\
\hline pitomba & Talisia esculenta (St. Hil.) Radlk & Sapindaceae & A & 9 \\
\hline cacto & $\begin{array}{c}\text { Nopalea cochenillifera Salm Dyck., } \\
\text { Ferocactus sp., }\end{array}$ & Cactaceae & $\mathrm{O}$ & 8 \\
\hline cana & Saccharum officinarum L. & Poaceae & A & 8 \\
\hline colônia & $\begin{array}{c}\text { Alpinia zerumbet (Pers.) B.L. Burtt } \\
\text { \& R.M. Sm. }\end{array}$ & Zingiberaceae & M & 8 \\
\hline erva-cidreira & Melissa officinalis L. & Lamiaceae & M & 8 \\
\hline antúrio & Anthurium andraeanum Lindl. & Araceae & $\mathrm{O}$ & 7 \\
\hline comigo-ninguém-pode & Dieffenbachia picta Schott. & Araceae & $\mathrm{O}$ & 7 \\
\hline hortência & Hydrangea macrophylla Ser., & Saxifragaceae & $\mathrm{O}$ & 7 \\
\hline jatobá & Hymenaea courbaril L. & Fabaceae & A, M & 7 \\
\hline poejo & Mentha pulegium L. & Lamiaceae & M & 7 \\
\hline abóbora & Cucurbita pepo L. & Cruciferae & A & 6 \\
\hline batata-doce & Ipomoea batatas (L.) Lam. & Convolvulaceae & A & 6 \\
\hline beijinho & Impatiens walleriana Hook. f. & Balsaminaceae & $\mathrm{O}$ & 6 \\
\hline espada-de-ogum & Sansevieria stuckyi God.-Leb & Rubiaceae & Ot & 6 \\
\hline guaco & Mikania glomerata Spreng. & Asteraceae & M & 6 \\
\hline jucá & Caesalpiniaceae ferrea Mart. & Caesalpiniaceae & M & 6 \\
\hline rosa & Rosa sp. & Rosaceae & $\mathrm{O}$ & 6 \\
\hline salsa & Petroselinum sativum Hoffm. & Apiaceae & A & 6 \\
\hline tamarino & Tamarindus indica St.-Hil. & Caesalpiniaceae & $\mathrm{A}, \mathrm{M}$ & 6 \\
\hline algodão & Gossypium hirsutum L. & Malvaceae & M & 5 \\
\hline
\end{tabular}




\begin{tabular}{|c|c|c|c|c|}
\hline Nome Popular & Nome Científico & Família & $\begin{array}{c}\text { Categoria } \\
\text { de Uso }\end{array}$ & NC \\
\hline anador & Justicia pectoralis Jacq. & Acanthaceae & $\mathrm{M}$ & 5 \\
\hline $\begin{array}{l}\text { cancerosa ou } \\
\text { espinheira-santa }\end{array}$ & Maytenus ilicifolia Mart. & Celastraceae & M & 5 \\
\hline carambola & Avehrroa carambola L. & Oxalidaceae & A & 5 \\
\hline dama-da-noite & $\begin{array}{l}\text { Epiphyllum oxipetalum (DC.) } \\
\text { Haworth }\end{array}$ & Cactaceae & $\mathrm{O}$ & 5 \\
\hline erva-de-bicho & $\begin{array}{c}\text { Polygonum persicaria var. } \\
\text { persicaria }\end{array}$ & Polygonaceae & M & 5 \\
\hline gerbão & $\begin{array}{c}\text { Stachytarpheta cayennensis (Rich.) } \\
\text { Vahl. }\end{array}$ & Verbenaceae & M & 5 \\
\hline jurubeba & Solanum paniculatum L. & Solanaceae & $\mathrm{A}, \mathrm{M}$ & 5 \\
\hline maracujá & Passiflora alata Curtis. & Passifloraceae & $\mathrm{A}, \mathrm{M}$ & 5 \\
\hline pitanga & Eugenia uniflora L. & Myrtaceae & A & 5 \\
\hline quebra pedra & Phyllanthus niruri L. & Dilleniaceae & $\mathrm{M}$ & 5 \\
\hline tangerina & Citrus reticulata Blanco & Rutaceae & A & 5 \\
\hline alecrim & Rosmarinus officinalis L. & Lamiaceae & M & 4 \\
\hline algodãozinho-do-cerrado & $\begin{array}{c}\text { Cochlospermum regium (Mart. \& } \\
\text { Sch.) Pilger }\end{array}$ & Cochlospermaceae & M & 4 \\
\hline cabaça & Lagenaria vulgaris Ser. & Cucurbitaceae & $\mathrm{O}$ & 4 \\
\hline caferana ou boldo-do-chile & Vernonia polyanthes L. & Compositae & M & 4 \\
\hline erva-de-santa-maria & Chenopodium ambrosioides L. & Chenopodiaceae & M & 4 \\
\hline gengibre & Zingiber officinale Roscol. & Zingiberaceae & M & 4 \\
\hline jenipapo & Genipa americana L. & Rubiaceae & Ot & 4 \\
\hline lima-da-pérsia & Citrus sp. & Rutaceae & $\mathrm{A}, \mathrm{M}$ & 4 \\
\hline mamona & Ricinus communis L. & Euphorbiaceae & M & 4 \\
\hline mexirica & Citrus nobilis Lour. & Rutaceae & A & 4 \\
\hline papoula & Papaver rhoeas L. & Papaveroideae & $\mathrm{O}$ & 4 \\
\hline pimentão & Capsicum annuum L. & Solanaceae & A & 4 \\
\hline sangra-d'água & Croton urucurana Baill. & Euphorbiaceae & M & 4 \\
\hline $\begin{array}{c}\text { santa-rita ou } \\
\text { flor-de-primavera ou } \\
\text { primavera ou buganvília }\end{array}$ & Bougainvillea glabra Choisy & Nyctaginaceae & $\mathrm{O}$ & 4 \\
\hline urucum & Bixa orellana L. & Bixaceae & M & 4 \\
\hline vick & Mentha arvensis L. & Lamiaceae & M & 4 \\
\hline alfavaca & Ocimum basilicum L. & Lamiaceae & M & 3 \\
\hline amora & Morus nigra L. & Moraceae & $\mathrm{A}, \mathrm{M}$ & 3 \\
\hline azaleia & Rhododendron simsii Planch. & Ericaceae & $\mathrm{O}$ & 3 \\
\hline castela (tipo de coentro) & S.I. & S.I. & A & 3 \\
\hline embaúba ou embaúva & Cecropia pachystachya Trèc. & Malvaceae & $\mathrm{M}, \mathrm{Ot}$ & 3 \\
\hline fedegoso & Senna occidentalis St.-Hil. & Caesalpiniaceae & M & 3 \\
\hline lírio & Lilium L. & Liliaceae & $\mathrm{O}$ & 3 \\
\hline lixeira & Curatella americana L. & Dilleniaceae & $\mathrm{M}$ & 3 \\
\hline manjerona (orégano) & Origanum majorana L. & Lamiaceae & M & 3 \\
\hline melão-de-são-caetano & Momordica charantia L. & Cucurbitaceae & M & 3 \\
\hline norvônica ou losna & Artemisia absinthium L. & Asteraceae & M & 3 \\
\hline pata-de-boi ou pata-de-vaca & Bauhinia nitida St.-Hil & Caesalpiniaceae & M & 3 \\
\hline picão & Bidens pilosa L. & Asteraceae & M & 3 \\
\hline quiabo & $\begin{array}{l}\text { Abelmoschus esculentus (L.) } \\
\text { Moench. }\end{array}$ & Malvaceae & A & 3 \\
\hline uva & Vitis L. & Vitaceae & A & 3 \\
\hline alface & Lactuca sativa $\mathrm{L}$. & Asteraceae & A & 2 \\
\hline amescla & $\begin{array}{l}\text { Protium heptaphyllum (Aubl.) } \\
\text { March. }\end{array}$ & Burseraceae & M & 2 \\
\hline angélica & Polianthes tuberosa L. & Agavaceae & $\mathrm{O}$ & 2 \\
\hline aroeira & Myracondruon urundeuva FR.All. & Anacardiaceae & M & 2 \\
\hline babaçu & Orbignya oleifera Burret. & Arecaceae & $\mathrm{O}$ & 2 \\
\hline boldo-miúdo & Plectrantus ornatus (Lour.) Spreng & Lamiaceae & M & 2 \\
\hline cajá & Spondias mombin L. & Anacardiaceae & A & 2 \\
\hline carrapicho & Acanthospermum sp. & Asteraceae & M & 2 \\
\hline
\end{tabular}




\begin{tabular}{|c|c|c|c|c|}
\hline Nome Popular & Nome Científico & Família & $\begin{array}{c}\text { Categoria } \\
\text { de Uso }\end{array}$ & NC \\
\hline carvão-branco & Callisthene fasciculata (Spr.) Mart. & Vochysiaceae & $\mathrm{M}$ & 2 \\
\hline chifre-de-veado & $\begin{array}{c}\text { Platycerium bifurcatum (Cav.) C. } \\
\text { Chr. }\end{array}$ & Polypodiaceae & $\mathrm{O}$ & 2 \\
\hline conta-de-leite & S.I. & S.I. & M & 2 \\
\hline coroinha & S.I. & S.I. & Ot & 2 \\
\hline erva-molar & Holcus lanatus L. & Poaceae & M & 2 \\
\hline eucalipto & Eucalyptus globulus Labill. & Myrtaceae & $\mathrm{M}$ & 2 \\
\hline feijão-andú & Cajanus cajan (L) Hunth. & Fabaceae & A & 2 \\
\hline figo & Ficus carica L. & Moraceae & A & 2 \\
\hline gonsaleiro & $\begin{array}{l}\text { Stronium fraxinifolium Schott ex } \\
\text { Spreng. }\end{array}$ & Anacardiaceae & M & 2 \\
\hline graviola & Annona muricata L. & Annonaceae & A & 2 \\
\hline ingá & Inga laurina (Sw.) Willd. Seeds. & Fabaceae & A & 2 \\
\hline ipê & Tabebuia sp. & Bignoniaceae & $\mathrm{O}$ & 2 \\
\hline mama-de-porca & Zanthoxylum rhoifolium & Rutaceae & $\mathrm{M}$ & 2 \\
\hline mangaba-brava & Lafoensia pacari St. Hil & Lythraceae & M & 2 \\
\hline manjericão & Ocimum sp. & Lamiaceae & A & 2 \\
\hline marmelada-de-espinho & S.I. & S.I. & Ot & 2 \\
\hline maxixe & Cucumis anguria $\mathrm{L}$. & Cucurbitaceae & A & 2 \\
\hline palmeira & S.I. & S.I. & $\mathrm{O}$ & 2 \\
\hline pepino & Cucumis satious L. & Cucurbitaceae & A & 2 \\
\hline pimenta-vermelha & Capsicum baccatum L. & Solanaceae & A & 2 \\
\hline Pinhão-roxo & Jatropha gossypiifolia L. & Euphorbiaceae & $\mathrm{M}$ & 2 \\
\hline pocã & Citrus reticulate $\mathrm{L}$. & Rutaceae & A & 2 \\
\hline quina & Strychnos pseudoquina A. St.-Hil. & Loganiaceae & $\mathrm{M}$ & 2 \\
\hline radite & Cichorium intybus L. & Asteraceae & A & 2 \\
\hline tarumã & Vitex cymosa Bert & Verbenaceae & M & 2 \\
\hline terramicina & $\begin{array}{c}\text { Alternanthera brasiliana (L.) } \\
\text { kuntze. }\end{array}$ & Amaranthaceae & M & 2 \\
\hline tomate & Lycopersium esculentum L. & Solanaceae & A & 2 \\
\hline uiti & Licania tomentosa (Benth.) Fritsch & Chrysobalanaceae & $\mathrm{Ot}$ & 2 \\
\hline violeta & Viola sp. & Violaceae & $\mathrm{O}$ & 2 \\
\hline açoita-cavalo & Luehea divaricata Mart. & Malvaceae & M & 1 \\
\hline acuri & $\begin{array}{l}\text { Phalerata Scheelea (Mart. ex } \\
\text { Spreng.) Burret }\end{array}$ & Arecaceae & A & 1 \\
\hline amburana & $\begin{array}{l}\text { Amburana cearenses (Freire } \\
\text { Allemão) A. C. Smith. }\end{array}$ & Fabaceae & Ot & 1 \\
\hline ampicilina-roxa & $\begin{array}{c}\text { Alternanthera brasiliana (L.) } \\
\text { Kuntze }\end{array}$ & Amaranthaceae & $\mathrm{M}$ & 1 \\
\hline artemisia & Artemisia anпиа L. & Asteraceae & M & 1 \\
\hline assa-peixe & Vernonia ferruginea Less. & Asteraceae & $\mathrm{M}$ & 1 \\
\hline atrativo & S.I. & S.I. & M & 1 \\
\hline beladona & Atropa belladonna L. & Solanaceae & $\mathrm{Ot}$ & 1 \\
\hline boldo-chinês & Plectrantus ornatus (Lour.) Spreng & Lamiaceae & M & 1 \\
\hline bromélia & $\begin{array}{c}\text { Alcantarea imperialis (Carrière) } \\
\text { Harms }\end{array}$ & Bromeliaceae & $\mathrm{O}$ & 1 \\
\hline cacau & Theobroma cacao L. & Malvaceae & A & 1 \\
\hline cainca & Chiococca anguicida Mart. & Rubiaceae & $\mathrm{M}$ & 1 \\
\hline cambará & Lantana camara $\mathrm{L}$. & Verbenaceae & $\mathrm{M}$ & 1 \\
\hline cambarú & Dipteryx alata Vog. & Fabaceae & M & 1 \\
\hline cânfora & $\begin{array}{c}\text { Cinnamomun comphora (L.) Nees } \\
\text { \& Eberm. }\end{array}$ & Lauraceae & $\mathrm{M}$ & 1 \\
\hline caruru-de-porco & Amaranthus viridis $\mathrm{L}$. & Amaranthaceae & $\mathrm{M}$ & 1 \\
\hline cavalinha & Equisetum giganteum $\mathrm{L}$. & Equisetaceae & $\mathrm{M}$ & 1 \\
\hline cereja & Prunus serrulata Lindl. & Rosaceae & $\mathrm{O}$ & 1 \\
\hline chagueira & S.I. & S.I. & Ot & 1 \\
\hline chama-dinheiro & S.I. & S.I. & Ot & 1 \\
\hline chapecanga & S.I. & S.I. & M & 1 \\
\hline
\end{tabular}




\begin{tabular}{|c|c|c|c|c|}
\hline Nome Popular & Nome Científico & Família & $\begin{array}{c}\text { Categoria } \\
\text { de Uso }\end{array}$ & NC \\
\hline chimbuva & Enterolobium timbouva Mart. & Fabaceae & $\mathrm{Ot}$ & 1 \\
\hline cinco-palma & S.I. & S.I. & $\mathrm{O}$ & 1 \\
\hline costela-de-adão & Monstera deliciosa Liebm. & Araceae & M & 1 \\
\hline couve & Brassica oleracea L. & Cruciferae & A & 1 \\
\hline cumbarú & Dipteryx alata Vog. & Fabaceae & M & 1 \\
\hline ecsória & Ixora coccinea L. & Rubiaceae & $\mathrm{O}$ & 1 \\
\hline elixir paregórico & Piper callosum Ruiz \&Pav. & Piperaceae & M & 1 \\
\hline erva-doce & Pimpinella anisum L. & Apiaceae & M & 1 \\
\hline espinho-de-touro & S.I. & S.I. & M & 1 \\
\hline espirradeira & Nerium oleander L. & Apocynaceae & $\mathrm{O}$ & 1 \\
\hline figueira & Ficus L. & Moraceae & $\mathrm{Ot}$ & 1 \\
\hline fortuna & $\begin{array}{c}\text { Bryophyllum pinnatum (Lam.) } \\
\text { Kurz }\end{array}$ & Crassulaceae & M & 1 \\
\hline fruta-de-veado & $\begin{array}{c}\text { Platycerium bifurcatum (Cav.) C. } \\
\text { Chr. }\end{array}$ & Polypodiaceae & $\mathrm{O}$ & 1 \\
\hline garirobinha & Syagrus oleracea (Mart.) Becc. & Arecaceae & $\mathrm{Ot}$ & 1 \\
\hline hortelanzinho & Hyptis cana Pohl ex Benth. & Lamiaceae & M & 1 \\
\hline ingá-de-metro & Inga laurina (Sw.) Willd. & Fabaceae & A & 1 \\
\hline jacinto & Hyacinthus L. & Asparagaceae & $\mathrm{O}$ & 1 \\
\hline jambo & $\begin{array}{c}\text { Sysygium malaccense (L.) Merr. \& } \\
\text { L.M. Perry }\end{array}$ & Myrtaceae & A & 1 \\
\hline jaquicendi & S.I. & S.I. & M & 1 \\
\hline jasmim & Jasminum officinale L. & Oleaceae & A & 1 \\
\hline jatobá-mirim & $\begin{array}{l}\text { Hymenaea coubarilL. var. } \\
\text { stilbocarpa (Hayne) Lee }\end{array}$ & Caesalpiniaceae & A & 1 \\
\hline jurema & $\begin{array}{c}\text { Chloroleucon acacioides (Ducke) } \\
\text { Barneby \& J.W.Grimes }\end{array}$ & Fabaceae & $\mathrm{Ot}$ & 1 \\
\hline laião & S.I. & S.I. & M & 1 \\
\hline laranja para doce & Citrus medica L. & Rutaceae & M & 1 \\
\hline levante & Mentha spicata L. & Lamiaceae & M & 1 \\
\hline malva & Malva sylvestris L. & Malvaceae & M & 1 \\
\hline malva-branca & Malva sylvestris L. & Malvaceae & M & 1 \\
\hline melancia & Citrullus lanatus (Thunb.) Mansf. & Cucurbitaceae & A & 1 \\
\hline melissa ou cidreira de folha & Melissa officinalis L. & Lamiaceae & M & 1 \\
\hline milho & Zea mays L. & Poaceae & A & 1 \\
\hline nil & S.I. & S.I. & M & 1 \\
\hline onze-horas & Portulaca grandiflora Hook. & Portulacaceae & $\mathrm{O}$ & 1 \\
\hline pachulin & Pogostemon patchouly Pellet. & Lamiaceae & $\mathrm{O}$ & 1 \\
\hline pau-de-bicho & Erminalia argentea Mart. & Combretaceae & $\mathrm{Ot}$ & 1 \\
\hline pau-brasil & Caesalpinia peltophoroides Benth. & Fabaceae & $\mathrm{Ot}$ & 1 \\
\hline pimenta-do-reino & Piper nigrum L. & Piperaceae & A & 1 \\
\hline pingo-de-ouro & Duranta repens aurea $\mathrm{L}$. & Verbenaceae & $\mathrm{O}$ & 1 \\
\hline rabo-de-raposa & $\begin{array}{c}\text { Arrojadoa rhodantha (Gürke) } \\
\text { Britton \& Rose }\end{array}$ & Cactaceae & $\mathrm{O}$ & 1 \\
\hline rosa branca & Rosa alba L. & Rosaceae & $\mathrm{O}$ & 1 \\
\hline rosa-do-deserto & $\begin{array}{c}\text { Adenium obesum (Forssk.) Roem. } \\
\text { \& Schult. }\end{array}$ & Apocynaceae & $\mathrm{O}$ & 1 \\
\hline rosamélia & S.I. & S.I. & $\mathrm{O}, \mathrm{M}$ & 1 \\
\hline rosquinha & S.I. & S.I. & M & 1 \\
\hline rúcula & Eruca sativa Mill. & Brassicaceae & A & 1 \\
\hline sabugueiro & Sambucus nigra L. & Caprifoliaceae & M & 1 \\
\hline tapera-velha & Hyptis suaveolens (L.) Poit. & Lamiaceae & M & 1 \\
\hline tomate-cereja & Lycopersicum sp. & Solanaceae & A & 1 \\
\hline vassourinha & Scoparia dulcis L. & Scrophulariacese & M & 1 \\
\hline
\end{tabular}

Legenda: NC: Número de Citações; A: Alimentar; M: Medicinal; O: Ornamental; Ot: Outros; S.I.: Sem Identificação. 
Do total dos informantes, $85 \%$ relataram que, além das plantas utilizadas como medicinais pertencentes aos quintais, eles retiram do cerrado e da mata ciliar alguns dos seus remédios. O depoente que mais nomeou espécies retiradas dessa unidade de paisagem com um total de 14 plantas medicinais foi do sexo feminino. Essas duas unidades de paisagem constituem a flora do Distrito Sucuri.

Pasa (2007) afirma que é de fundamental importância coletar junto à população informações a respeito do uso de plantas, ressalta também que as plantas medicinais estão sendo revalorizadas porque, entre outras razões, é a forma mais acessível para a população local curar suas enfermidades.

A Tabela 2 registra as 54 espécies medicinais usadas pelos depoentes da comunidade, bem como a forma de uso e as finalidades de usos, através do conhecimento empírico da população local. Foram identifi- cadas 31 famílias botânicas com um total de 148 citações. Hymenaea courbaril L. (jatobá) e o Strychnos pseudoquina A. St.-Hil. (quina) foram as mais citadas, ambas com $8,10 \%$. Metade das espécies foi citada uma única vez com o total 21. A parte da planta mais utilizada foi a entrecasca com $45 \%$, em segundo lugar estão as folhas com $28 \%$, raiz com $15 \%$ e a categoria outros com aproximadamente $12 \%$ incluindo fruto, semente, planta inteira, vagem, resina e broto. A forma de preparo que recebeu destaque com quase $30 \%$ foi o xarope, seguido do chá com $27 \%$, e a categoria outros com $16,5 \%$, seguido de banho de assento e maceração com $7 \%$, infusão com $5 \%$, garrafada com $3 \%$, torrada e queimada com $2,5 \%$ e serenada com $1 \%$. A categoria outros está representada por compressa, emplastro, curtido no vinho ou na água, fervido ou descansado no leite, batido no leite ou na água com sal e outras formas.

Tabela 2 - Espécies medicinais usadas na Comunidade Sucuri, Cuiabá, MT. 2014.

\begin{tabular}{|c|c|c|c|c|c|}
\hline Nome Popular & Nome Científico & $\begin{array}{c}\text { Parte } \\
\text { Utilizada }\end{array}$ & Forma de Uso & Indicação & $\mathrm{NC}$ \\
\hline jatobá & Hymenaea courbaril L. & $\begin{array}{l}\text { entrecasca e } \\
\text { fruto }\end{array}$ & $\begin{array}{l}\text { xarope, curtido no } \\
\text { vinho branco e chá }\end{array}$ & $\begin{array}{c}\text { sistema respiratório, } \\
\text { anemia, anti-inflamató- } \\
\text { rio, bom para tudo }\end{array}$ & 12 \\
\hline quina & $\begin{array}{c}\text { Strychnos pseudoquina } \\
\text { A. St.-Hil. }\end{array}$ & entrecasca & $\begin{array}{c}\text { xarope, } \\
\text { chá, torrada, } \\
\text { serenada, queimad } \\
\text { e curtida na água }\end{array}$ & $\begin{array}{c}\text { vermífugo, anemia, âni- } \\
\text { mo na criança, sistema } \\
\text { digestório }\end{array}$ & 12 \\
\hline aroeira & $\begin{array}{l}\text { Myracrodruon urun- } \\
\text { deuva Fr. All. }\end{array}$ & $\begin{array}{l}\text { entrecasca, } \\
\text { folhas e } \\
\text { resina }\end{array}$ & $\begin{array}{c}\text { emplasto, chá e } \\
\text { xarope }\end{array}$ & $\begin{array}{l}\text { sistema respiratório, } \\
\text { cicatrização, hematoma } \\
\text { e anti-inflamatório }\end{array}$ & 8 \\
\hline cambará & Lantana cal & $\begin{array}{l}\text { entrecasca e } \\
\text { folhas }\end{array}$ & xarope & sistema respiratório & 7 \\
\hline jucá & $\begin{array}{c}\text { Caesalpiniaceae ferrea } \\
\text { Mart. }\end{array}$ & vagem & $\begin{array}{l}\text { curtido no vinho, } \\
\text { cozido, chá, banho } \\
\text { e macerado }\end{array}$ & $\begin{array}{c}\text { anti-inflamatório, cica- } \\
\text { trizante, emagrecedor, } \\
\text { sistema digestório e } \\
\text { diabetes }\end{array}$ & 6 \\
\hline $\begin{array}{l}\text { algodão-do- } \\
\text { cerrado }\end{array}$ & $\begin{array}{l}\text { Cochlospermum regium } \\
\text { (Schrank) pilg. }\end{array}$ & Eolhas & $\begin{array}{c}\text { chá, batido com } \\
\text { água e sal e garra- } \\
\text { fada }\end{array}$ & $\begin{array}{c}\text { aparelho reprodutor } \\
\text { feminino, sistema circu- } \\
\text { latório }\end{array}$ & 5 \\
\hline $\begin{array}{l}\text { hortelã-do- } \\
\text { campo }\end{array}$ & $\begin{array}{l}\text { Hyptis cana Pohl ex } \\
\text { Benth. }\end{array}$ & $\begin{array}{c}\text { folhas e } \\
\text { entrecasca }\end{array}$ & $\begin{array}{l}\text { chá, torrada e xa- } \\
\text { rope }\end{array}$ & $\begin{array}{l}\text { sistema digestório, } \\
\text { sistema respiratório, } \\
\text { vermífugo }\end{array}$ & 5 \\
\hline quebra-pedra & Phyllanthus niruri L. & folhas e raiz & chá e infusão & sistema urinário & 5 \\
\hline tap & $\begin{array}{l}\text { Hyptis suaveolens (L.) } \\
\text { Poit. }\end{array}$ & $\begin{array}{l}\text { planta in- } \\
\text { teira }\end{array}$ & $\begin{array}{c}\text { chá, xarope e in- } \\
\text { fusão }\end{array}$ & $\begin{array}{l}\text { dor de cab } \\
\text { digestóri }\end{array}$ & 5 \\
\hline $\begin{array}{l}\text { para-tudo- do- } \\
\text { mato }\end{array}$ & $\begin{array}{l}\text { Tabebuia aurea (Cha- } \\
\text { miso) Sandwith }\end{array}$ & entrecasca & $\begin{array}{l}\text { xarope e fervido no } \\
\text { leite }\end{array}$ & anemia, vermífugo & 5 \\
\hline amescla & $\begin{array}{l}\text { Protium heptaphyllum } \\
\text { (Aubl.) March. }\end{array}$ & entrecasca & xarope & sistema respiratório & 4 \\
\hline $\begin{array}{l}\text { melão-c } \\
\text { caet }\end{array}$ & Momordica & $\begin{array}{l}\text { pó e fo- } \\
\text { lhas }\end{array}$ & $\begin{array}{l}\text { chá, banho e mace- } \\
\text { rado }\end{array}$ & $\begin{array}{l}\text { dengue, malária, fungo } \\
\text { no couro cabeludo, he- } \\
\text { morroida }\end{array}$ & 4 \\
\hline
\end{tabular}




\begin{tabular}{|c|c|c|c|c|c|}
\hline Nome Popular & Nome Científico & $\begin{array}{c}\text { Parte } \\
\text { Utilizada }\end{array}$ & Forma de Uso & Indicação & NC \\
\hline negramina & $\begin{array}{l}\text { Siparuna guianen- } \\
\text { sis Aublet. }\end{array}$ & folhas & banho e chá & $\begin{array}{l}\text { dor de cabeça, mal } \\
\text { estar, sistema respirató- } \\
\text { rio, quebrante }\end{array}$ & 4 \\
\hline angico & $\begin{array}{l}\text { Anadenanthera falcata } \\
\text { (Benth.) Speg. }\end{array}$ & entrecasca & xarope & sistema respiratório & 3 \\
\hline barbatimão & $\begin{array}{l}\text { Stryphnodendron ads- } \\
\text { tringens (Mart.) Coville }\end{array}$ & entrecasca & $\begin{array}{c}\text { curtido na água e } \\
\text { chá }\end{array}$ & $\begin{array}{c}\text { aparelho reprodutor } \\
\text { feminino, cicatrização, } \\
\text { coceira }\end{array}$ & 3 \\
\hline cajazinho & Spondias mombin L. & $\begin{array}{c}\text { entrecasca e } \\
\text { raiz }\end{array}$ & macerado & diabetes & 3 \\
\hline erva-de-bicho & $\begin{array}{l}\text { Polygonum persicaria } \\
\text { var. persicaria }\end{array}$ & folhas & banho e xarope & sistema respiratório & 3 \\
\hline jequitibá & $\begin{array}{c}\text { Cariniana legalis Kunt- } \\
\text { ze. }\end{array}$ & entrecasca & $\begin{array}{l}\text { banho e banho de } \\
\text { assento }\end{array}$ & $\begin{array}{l}\text { aparelho reprodutor } \\
\text { feminino, dor na coluna }\end{array}$ & 3 \\
\hline nó-de-cachorro & $\begin{array}{l}\text { Heteropteris aphrodisia- } \\
\text { ca O. Mach. }\end{array}$ & raiz & macerado & anti-inflamatório & 3 \\
\hline tarumã & Vitex cymosa Bert & $\begin{array}{c}\text { folhas e } \\
\text { entrecasca }\end{array}$ & chá & dor de barriga, diarreia & 3 \\
\hline assa-peixe & $\begin{array}{c}\text { Vernonia ferruginea } \\
\text { Less. }\end{array}$ & raiz & xarope & sistema respiratório & 2 \\
\hline crista-de-galo & Celosia cristata L. & folhas & pó da folha e chá & $\begin{array}{l}\text { cicatrização, câncer, sis- } \\
\text { tema urinário, coluna }\end{array}$ & 2 \\
\hline cumbaru & Dipteryx alata Vog. & entrecasca & chá & diarreia & 2 \\
\hline $\begin{array}{c}\text { erva-de-santa- } \\
\text { maria }\end{array}$ & $\begin{array}{c}\text { Chenopodium ambrosio- } \\
\text { ides L. }\end{array}$ & folhas & xar & vermífugo & 2 \\
\hline erva-molar & Holcus lanatus L. & folhas & chá & sistema urinário & 2 \\
\hline espinheiro & $\begin{array}{c}\text { Crataegus Oxyacan- } \\
\text { tha L. }\end{array}$ & entrecasca & xarope & sistema respiratório & 2 \\
\hline gerbão & $\begin{array}{l}\text { Stachytarpheta cayen- } \\
\text { nensis (Rich.)Vahl. }\end{array}$ & raiz & xarope e infusão & $\begin{array}{c}\text { sistema digestório, } \\
\text { diarreia }\end{array}$ & 2 \\
\hline jatobá-mirim & $\begin{array}{l}\text { Hymenaea coubarilL. } \\
\text { var. stilbocarpa } \\
\text { (Hayne) Lee }\end{array}$ & entrecasca & xarope & sistema respiratório & 2 \\
\hline lixeira & Curatella americana L. & folhas & chá & emagrece & 2 \\
\hline $\begin{array}{l}\text { mangava-bra- } \\
\text { va (piúna) }\end{array}$ & Lafoensia pacari St. Hil & entrecasca & $\begin{array}{c}\text { curtido na água e } \\
\text { chá }\end{array}$ & sistema digestório & 2 \\
\hline pequi & $\begin{array}{c}\text { Caryocar brasiliense } \\
\text { Camb. }\end{array}$ & folhas secas & infusão e chá & $\begin{array}{l}\text { sistema urinário, co- } \\
\text { luna }\end{array}$ & 2 \\
\hline vassorinha & Scoparia dulcis L. & $\begin{array}{l}\text { planta in- } \\
\text { teira }\end{array}$ & chá e compressa & sistema urinário, torsão & 2 \\
\hline $\begin{array}{l}\text { alecrim-do- } \\
\text { mato }\end{array}$ & $\begin{array}{c}\text { Baccharis caprariifolia } \\
\text { DC. }\end{array}$ & folhas & garrafada & $\begin{array}{l}\text { aparelho reprodutor } \\
\text { feminino }\end{array}$ & 1 \\
\hline amora & Morus alba L. & folhas & infusão & reposição hormonal & 1 \\
\hline $\begin{array}{l}\text { arnica (picão } \\
\text { branco) }\end{array}$ & Arnica montana L. & raiz & infusão & hepatite & 1 \\
\hline $\begin{array}{l}\text { aspirina-do- } \\
\text { campo }\end{array}$ & S.I. & raiz & chá & febre & 1 \\
\hline bocaiuva & $\begin{array}{l}\text { Acrocomia aculeata } \\
\text { (Jacq.) Lodd. Ex mart. }\end{array}$ & fruto & batido com leite & anemia & 1 \\
\hline caiapia & $\begin{array}{c}\text { Dorstenia Multiformis } \\
\text { Miq. }\end{array}$ & batata (raiz) & garrafada & $\begin{array}{l}\text { aparelho reprodutor } \\
\text { feminino }\end{array}$ & 1 \\
\hline caju-amarelo & $\begin{array}{c}\text { Anacardium occidentale } \\
\text { L. }\end{array}$ & fruto & comer & diabetes & 1 \\
\hline $\begin{array}{l}\text { cajuzinho-do- } \\
\text { campo }\end{array}$ & $\begin{array}{c}\text { Anacardium humile A. } \\
\text { St.-Hil }\end{array}$ & raiz & garrafada & reumatismo & 1 \\
\hline calunga & $\begin{array}{c}\text { Simaba ferruginea A. } \\
\text { St.-Hil. }\end{array}$ & raiz & curtido na água & gordura do fígado & 1 \\
\hline $\begin{array}{l}\text { caninha-do- } \\
\text { brejo }\end{array}$ & $\begin{array}{c}\text { Costus spicatus (Jacq.) } \\
\text { Sw. }\end{array}$ & folhas & chá & sistema urinário & 1 \\
\hline dorme-dorme & Mimosa pudica L. & folhas & banho & criança dormir & 1 \\
\hline
\end{tabular}




\begin{tabular}{|c|c|c|c|c|c|}
\hline Nome Popular & Nome Científico & $\begin{array}{c}\text { Parte } \\
\text { Utilizada }\end{array}$ & Forma de Uso & Indicação & $\mathrm{NC}$ \\
\hline embaúba & $\begin{array}{c}\text { Cecropia pachystachya } \\
\text { Trèc. }\end{array}$ & broto & xarope & sistema respiratório & 1 \\
\hline fedegoso & $\begin{array}{c}\text { Senna occidentalis St.- } \\
\text { Hil. }\end{array}$ & folhas & curtido na pinga & sistema digestório & 1 \\
\hline $\begin{array}{l}\text { genciana ( } 5 \\
\text { folhas) }\end{array}$ & Genciana Lutea L. & raiz & queimada & $\begin{array}{l}\text { dor de cabeça, mens- } \\
\text { truação }\end{array}$ & 1 \\
\hline goiaba-branca & Psidium guajava L. & broto & chá & disenteria & 1 \\
\hline gonsaleiro & $\begin{array}{l}\text { Stronium fraxinifolium } \\
\text { Schott ex Spreng. }\end{array}$ & entrecasca & xarope & sistema respiratório & 1 \\
\hline ipê-roxo & $\begin{array}{l}\text { Tabebuia impetiginosa } \\
\text { (Mart.ex DC.) Standl. }\end{array}$ & entrecasca & chá & $\begin{array}{l}\text { limpa o organismo, } \\
\text { abre o apetite }\end{array}$ & 1 \\
\hline mastruz & $\begin{array}{l}\text { Chemopodium ambro- } \\
\text { sioides L. }\end{array}$ & folhas & curtido no leite & vermífugo & 1 \\
\hline picão & Bidens pilosa L. & raiz & infusão & hepatite & 1 \\
\hline sangra-d'água & Croton urucurana Baill. & $\begin{array}{l}\text { sangue (sei- } \\
\text { va) }\end{array}$ & $\begin{array}{c}1 \text { gota de sangue }+ \\
\text { água }\end{array}$ & câncer & 1 \\
\hline $\begin{array}{c}\text { saúde-da- } \\
\text { mulher }\end{array}$ & S.I. & batata (raiz) & garrafada & $\begin{array}{l}\text { aparelho reprodutor } \\
\text { feminino }\end{array}$ & 1 \\
\hline
\end{tabular}

Legenda: NC: Número de Citações; S.I.: Sem Identificação.

As indicações mais citadas foram para as patologias do Sistema Respiratório com 22\%, seguidas do Aparelho Digestório com 11\%; na sequência, as doenças do Sistema Urinário com $8 \%$, o aparelho reprodutor feminino e os vermífugos com $7,5 \%$ cada um, os indicados para cicatrização ocuparam o quinto lugar com $6 \%$, os anti-inflamatórios totalizaram $4,5 \%$, os menos citados foram anemia, diarreia e dor de barriga com $4,0 \%$, diabetes com $3,5 \%$ e Sistema Circulatório com 1,5\%. A categoria outros agrupou indicações para câncer, febre, dor de cabeça, apetite, limpa o organismo, criança dormir melhor, torção, dor na coluna, emagrecer, reumatismo entre outras com $19 \%$ de um total de 197 indicações conforme a classificação de doença segundo a Organização Mundial de Saúde, CID-10 (OMS, 2000).

\section{Considerações finais}

A importância do estudo etnobotânico realizado na comunidade Sucuri, que é considerada tradicional e em fase de aculturação, nos permite aproximar da realidade dessas populações resgatando o que elas possuem de melhor, que é o seu conhecimento de vida adquirido por décadas de experiências empíricas com a natureza.

Esse resgate do saber local serve de base para muitos estudos científicos que acabam testando e confirmando o que esses povos já utilizam na prática por muitos anos.
$\mathrm{Na}$ comunidade local, as pessoas locais praticam a conversação da biodiversidade mantendo assim a sustentabilidade ambiental referente às comunidades vegetais, como fonte permanente de uso diário para às diferentes etnocategorias botânicas locais.

\section{Referências}

ALMEIDA, Suzanne Estéfanie; PASA, Maria Corette; GUARIM, Vera Lucia Monteiro Santos (in memoriam). Uso da biodiversidade em quintais de comunidades tradicionais da Baía de Chacorore, Barão de Melgaço, Mato Grosso, Brasil. Biodiversidade, v. 13, n. 1, p. 141-155, 2014.

AMOROZO, Maria Christina Mello. A abordagem etnobotânica na pesquisa de plantas medicinais. In: DI STASI, L. C. (Org.). Plantas medicinais: arte e ciência, um guia de estudo interdisciplinar. São Paulo: EDUSP, 1996. p. 47-68.

AMOROZO, Maria Christina Mello. Os quintais funções, importância e futuro. In: GUARIN NETO, Germano; CARNIELLO, Maria Antônia (Org.). Quintais mato grossenses: espaços de conservação e reprodução de saberes. Cárceres, MT: Editora Unemat, 2008.

ANGIOSPERM PHYLOGENY GROUP - APG III. An update of the Angiosperm Phylogeny Group classification for the orders and families of flowering plants: APG III. Botanical Journal of the Linnean Society, 161: 105-121, 2009. Disponível em: <http:/ / onlinelibrary.wiley.com/ doi/10.1111/j.1095-8339.2009.00996.x/epdf>. Acesso em: 7 jan. 2015.

CABALLERO, Javier. La etnobotânica. In: BARRER, A. (Ed.). La etnobotânica: tres puntos de vista y uma perspectiva. Xalapa: Instituto de Investigación sobre Recursos Bióticos, 1979. p. 27-30.

COSTA, Gilmar da; SILVA, Patrícia Sanches da. Tratamento bioenergético: estudo etnofarmacológico de 
plantas medicinais da Pastoral da Saúde Alternativa de Cotriguaçú, MT. Biodiversidade, v. 13, n. 1, p. 115124, 2014. Disponível em: <http://periodicoscientificos.ufmt.br/ojs/index.php/biodiversidade/article/ view/1544/1214>. Acesso em: 7 fev. 2015.

DE DAVI, Margô; PASA, Maria Corette. As plantas medicinais e a etnobotânica em Várzea Grande, MT, Brasil. Interações, Campo Grande, MS, v. 16, n. 1, p. 97108, jan./jun. 2015.

INSTITUTO BRASILEIRO DE GEOGRAFIA E ESTATÍSTICA - IBGE. Censo Demográfico. Rio de Janeiro: IBGE, 2010. Disponível em: <http://www.censo2010.ibge. gov.br/sinopse $/$ index.php?uf $=51 \&$ dados $=6>$. Acesso em: 17 mar. 2015.

JOHNSON, Martha C. Lore-Capturing Traditional Environmental Knowledge. Ottawa, CA: Diane Publishing, 1992. 190p.

MINAYO, Maria Cecília de Souza. O desafio do conhecimento: pesquisa qualitativa em saúde. 10. ed. São Paulo: Hucitec, 2007.

MINAYO, Maria Cecília de Souza (Org.). Pesquisa social: teoria, método e criatividade. Petrópolis, RJ: Vozes, 1994.

ORGANIZAÇÃO MUNDIAL DE SAÚDE - OMS. CID - 10: Classificação Estatística Internacional de doenças e problemas relacionados à saúde. 10. ed. São Paulo: EDUSP, 2000. 354p.

PASA, Maria Corette. Etnobiologia de uma comunidade ribeirinha no alto da bacia do rio Aricá-Açú, Cuiabá, Mato Grosso, Brasil. 2004. 174f. Tese (Doutorado em Ciências) Centro de Ciências Biológicas e da Saúde, Universidade Federal de São Carlos, São Carlos, SP.

Um olhar etnobotânico sobre as comunidades do Bambá, Cuiabá, MT. Cuiabá, MT: Entrelinhas, 2007.

Abordagem etnobotânica na Comunidade de Conceição-Açú, Mato Grosso, Brasil. Polibotânica, México, v. 31, p. 169-197, 2011.

SIVIERO, Amauri; DELUNARDO, Thiago Andrés; HAVERROTH, Moacir; OLIVEIRA, Luis Claudio de; ROMAN, André Luis Cote; MENDONÇA, Ângela Maria da Silva. Plantas ornamentais em quintais urbanos de Rio Branco, Brasil. Bol. Mus. Para. Emílio Goeldi. Cienc. Hum., Belém, v. 9, n. 3, p. 797-813, set./dez. 2014. Disponível em: <http://www.museu-goeldi.br/editora/ bh/artigos/chv9n3_2014/plantas\%28siviero\%29.pdf>. Acesso em: 9 mar. 2015.

WORLD HEALTH ORGANIZATION - WHO. Who handbook for reporting results for cancer treatment. Geneva: World Health Organization, 1979. 